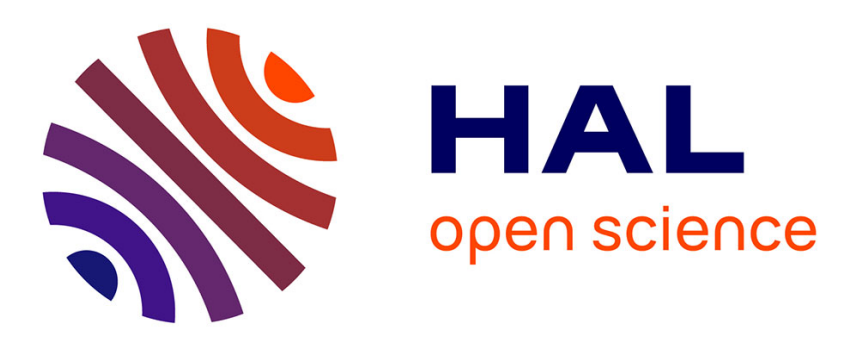

\title{
Broadband permeability measurement method for ferrites at any magnetization state: Experimental results
}

Alexis Chevalier, July Cortes, Jorge Lezaca, Patrick Queffelec

\section{To cite this version:}

Alexis Chevalier, July Cortes, Jorge Lezaca, Patrick Queffelec. Broadband permeability measurement method for ferrites at any magnetization state: Experimental results. Journal of Applied Physics, 2013, Vol. 114 (174904), pp.174904-1-6. 10.1063/1.4828876 . hal-00951692

\section{HAL Id: hal-00951692 \\ https://hal.univ-brest.fr/hal-00951692}

Submitted on 25 Feb 2014

HAL is a multi-disciplinary open access archive for the deposit and dissemination of scientific research documents, whether they are published or not. The documents may come from teaching and research institutions in France or abroad, or from public or private research centers.
L'archive ouverte pluridisciplinaire HAL, est destinée au dépôt et à la diffusion de documents scientifiques de niveau recherche, publiés ou non, émanant des établissements d'enseignement et de recherche français ou étrangers, des laboratoires publics ou privés. 


\title{
Broadband permeability measurement method for ferrites at any magnetization state: Experimental results
}

\author{
A. Chevalier, J. Cortes, J. Lezaca, and P. Queffelec \\ Laboratoire des Sciences et Techniques de l'information, de la Communication et de la Connaissance \\ (Lab-STICC) UMR CNRS 6285, European University of Brittany-UBO, CS 93837, 29238 Brest, France
}

(Received 12 July 2013; accepted 21 October 2013; published online 4 November 2013)

\begin{abstract}
This paper describes the experimental results obtained by a broadband permeability measurement method based on the full-wave electromagnetic (EM) analysis of a non-reciprocal transmission line. The method offers a new experimental tool for measuring the broadband dynamic behavior of ferrites whatever their magnetization state. The methodology and experimental setup are presented with the aim of extracting both the permittivity and the two components (diagonal $\mu$ and off-diagonal $\kappa$ ) of the permeability tensor. Experimental data on commercial ferrites set in different magnetization states are presented and discussed. Furthermore, this method opens perspectives for the determination of other useful magnetic parameters such as resonance linewidth $\Delta \mathrm{H}$. This quantity can be then measured at different frequencies, where conventional resonant methods give a value at a fixed frequency. (C) 2013 AIP Publishing LLC. [http://dx.doi.org/10.1063/1.4828876]
\end{abstract}

\section{INTRODUCTION}

Magnetic materials, especially ferrites, are widely used in microwave devices such as insulators, phase-shifters, and circulators because of the anisotropic nature of their electromagnetic (EM) properties. This anisotropy can be obtained by applying a static magnetic field to the material in order to magnetize it. Then the permeability of the material becomes a tensor in which the diagonal and off-diagonal components depend on both the frequency and the magnetization state. Thus the design of ferrite-based microwave devices requires the characterization of the dynamic properties of the material, i.e., the permittivity and the permeability. Unfortunately the experimental measurements of these EM properties are often limited to the demagnetized state (coaxial-line based method) or to the saturated state (resonance linewidth measurement using a resonant-cavity method). ${ }^{1}$ These two special cases are not sufficient to correctly describe the dynamic behavior of ferrite materials set in their EM environment for various microwave applications. Indeed, many applications need for partially magnetized materials such as tunable filters or self-biased circulators. And even for conventional circulators that used saturated ferrites, it was shown, through magnetostatic analysis, that some regions of the ferrite sample are partially magnetized due to the non-uniformity of the internal dc biasing field. In such a context, we have developed a broad-band characterization method which allows measuring the components of the permeability tensor (diagonal term $\mu$ and off-diagonal term $\kappa$ ) at any magnetization states of the material. This paper first presents the principle of the method, the second part describes the measurement cell and the experimental setup and the third part presents measurement results performed using a commercial ferrite sample showing well-known static magnetic properties.

\section{DESCRIPTION OF THE MEASUREMENT METHOD}

The method we have worked out is based on the measurement of the scattering parameters (S-parameters) of a strip transmission line partially filled with the magnetic material to be characterized. ${ }^{2}$ As shown in Figure 1, two identical slabs of the magnetic sample are set above and below the strip conductor. At each side of the strip two different dielectrics are placed (respective permittivities $\varepsilon_{1}$ and $\varepsilon_{2}$ ) to insure the non-reciprocal behavior $\left(\mathrm{S}_{21} \neq \mathrm{S}_{12}\right)$ of the line when the material under test is magnetized. This is necessary to obtain a sufficient number of measured complex parameters $\left(S_{21}, S_{12}, S_{11}\right)$ to be able to retrieve the three complex EM parameters of the material under test: the complex permittivity $\varepsilon$, the complex diagonal term $\mu$, and the complex off-diagonal term $\kappa$ of the permeability tensor. For a better understanding of the analysis of the measurement cell, we define the region of the line that is filled with the magnetic and dielectric slabs as the loaded section and the region before and after the loaded section as the air sections. The data processing program that we have developed to retrieve the constitutive parameters $\varepsilon, \mu$, and $\kappa$ is divided into two main stages.

The first stage is the "direct problem" in which the theoretical S-parameters of the line are determined as a function of the electromagnetic properties of materials in the cell and their geometrical dimensions. We performed an EM fullwave analysis of the measurement cell taking into account the contributions of the quasi-TEM dominant mode and higher order modes which are excited at the discontinuities between the loaded section and the air sections. For this analysis, we have proposed an equivalent model of the loaded section based on two assumptions. First, considering the strip's width "a" is highly greater than the distance from the strip to the ground plane "b," we can conclude that the energy in the line is mostly concentrated between the strip conductor and the ground planes. Second, due to the symmetry of the discontinuities only the $\mathrm{TE}_{\mathrm{n} 0}$ higher order modes are going to be excited in the cell. ${ }^{3}$

Figure 2 depicts the equivalent model matching only one half cross section due to the symmetry of the structure. We use Perfect Electric Conductor (PEC) to describe metallic central strip as well as ground planes and we use Perfect 


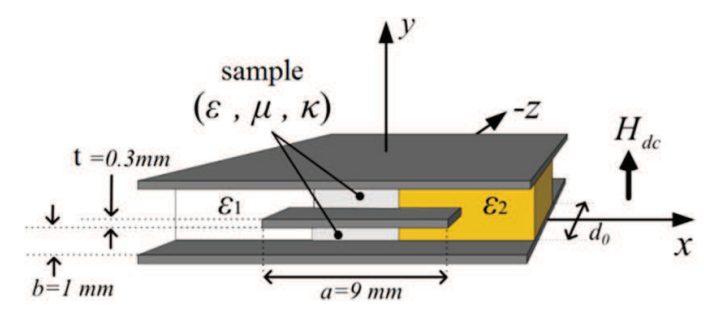

FIG. 1. Asymmetrical measurement cell.

Magnetic Conductors (PMC) at the edge of the strip in accordance with the EM field pattern. The full-wave analysis consists in determining the dispersion diagram of the existing modes in the different sections of the cell. ${ }^{4}$ In the operating frequency band [ $100 \mathrm{MHz}-12 \mathrm{GHz}$ ], the dispersion diagram of air sections is easily obtained from analytical relations as the propagation occurs only in the air. On the other hand, in the loaded section three materials are present (dielectric 1 , ferrite under test, and dielectric 2), the dispersion relation is more difficult to obtain. The EM components in each material are calculated based on their permittivity and permeability and continuity conditions are forced in both interfaces $\left(x=x_{1}\right.$, and $\mathrm{x}=\mathrm{x}_{2}$ ). Then a mode matching method is used in the z-direction to obtain theoretical S parameters. Details of this direct problem are presented in Ref. 4. To meet the assumptions used on the equivalent model $(\mathrm{a} \gg \mathrm{b})$, the design of the cell has led to a conductor width "a" equal to $9 \mathrm{~mm}$ and a height " $b$ " equal to $1 \mathrm{~mm}$ leading to a characteristic impedance of the stripline close to $18 \Omega$ which is away from the usual value of $50 \Omega$. We will have to make a special calibration procedure in the experimental setup to take this impedance mismatch into account. Finally, the typical width of the sample (L-h) is equal to $5 \mathrm{~mm}$.

The second stage is the "inverse problem": the aim is to find the electromagnetic parameters of the unknown material from S-parameter measurements. As there are no direct analytical relationships between S-parameters and constitutive parameters $\varepsilon, \mu$, and $\kappa$, a numerical optimization procedure is required to match theoretical $S$-parameters to the measured ones. The originality of our method is the use of a wideband optimization procedure in which the frequency spectra of $\varepsilon$, $\mu$, and $\kappa$ of the material under test are optimized. Instead of solving the inverse problem frequency by frequency as usually done, we have chosen an optimization simultaneously over all the operating frequency ranges by matching only the S-parameters modules. This optimization overcomes the uncertainty problems related to the phases of reflection parameters that arise around dimensional resonance frequencies where $\left|S_{i i}\right|(i=1,2)$ tend to zero.

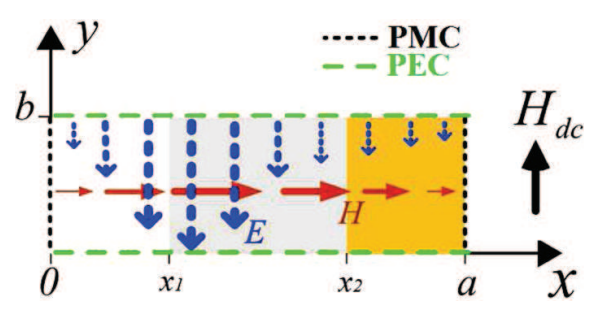

FIG. 2. Equivalent representation of the cross section of the cell.
In order to start this optimization procedure, we have to previously make a choice for the type of function of the frequency for each constitutive parameters $\varepsilon, \mu$, and $\kappa$. We have chosen to represent the dielectric properties of the material to be characterized by a dispersive permittivity function $\varepsilon(\mathrm{f})$ given by the Debye model. This model function is defined by three parameters $\varepsilon_{\infty}, \varepsilon_{\text {stat }}$, and $\tau$, where the relaxation time $\tau$ is very small in order to reject the relaxation very far in high frequency and the infinite permittivity $\varepsilon_{\infty}$ is slightly lower than the static permittivity $\varepsilon_{\text {stat }}$. For the permeability, we selected a physical model that describes the frequency dependence of the two components $\mu(\mathrm{f})$ and $\kappa(\mathrm{f})$. This model named "Generalized Permeability Tensor" ${ }^{, 5}$ was developed in our laboratory to describe the dynamic magnetic properties of polycrystalline ferrites at any magnetization state. The five input parameters of the model are the physical properties of the material: its saturation magnetization $4 \pi \mathrm{M}_{\mathrm{s}}$, its anisotropy field $\mathrm{H}_{\mathrm{a}}$, its damping factor $\alpha$, the applied static biasing field $\mathrm{H}_{\mathrm{dc}}$, and the demagnetizing factor $\mathrm{N}_{\mathrm{y}}$ in the direction of $\mathrm{H}_{\mathrm{dc}}$. Finally, the error function in the optimization procedure is defined by

$$
\begin{aligned}
F(X) & =\sum_{f \min }^{f \max }\left[\sum_{i=1}^{i=2} \sum_{j=1}^{j=2}\left(\left|S_{i j}^{(\text {théo })}(X, f)\right|-\left|S_{i j}^{(\text {mes })}(X, f)\right|\right)^{2}\right] \quad \text { where } \\
X & =[\varepsilon(f), \mu(f), \kappa(f)] .
\end{aligned}
$$

The eight physical parameters (three for the permittivity spectrum and five for the permeability tensor components spectra) of the models are the input variables of the optimization vector $X$. Once the minimal error is found, the theoretical S-parameters match the measured ones and therefore functions $\varepsilon(\mathrm{f}), \mu(\mathrm{f})$, and $\kappa(\mathrm{f})$ match the measured effective parameters of the material under test. We have developed a local code in Matlab® for these two stages.

\section{EXPERIMENTAL SETUP}

The measurement cell is built in brass; the geometrical dimensions are specified in Figure 3. Two SMA connectors are used to connect the cell to the junction cables of a network analyzer. In the propagation direction, we can identify several sections in the cell: A $50 \Omega$ coaxial section at the SMA connectors followed by a "taper" acting as the coaxial to stripline transition and finally $18 \Omega$ impedance stripline section. The four S-parameters are measured in the frequency band $[100 \mathrm{MHz}-12 \mathrm{GHz}$, thanks to a Vector Network Analyzer Agilent E8364A.

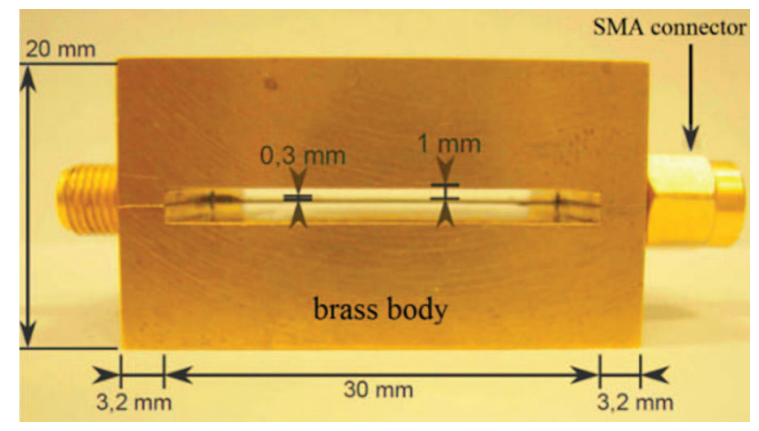

FIG. 3. Measurement cell (side view). 


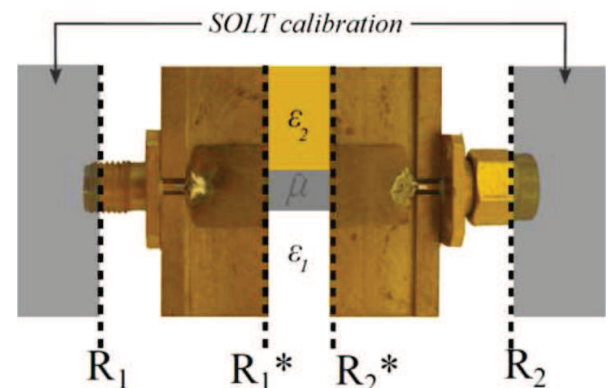

FIG. 4. Connector reference planes $\mathrm{R}_{1}, \mathrm{R}_{2}$; sample reference planes $\mathrm{R}_{1}$ *, $\mathrm{R}_{2}$ * (top view).

The first step of the experimental setup consists in calibrating the network analyzer in order to correct the systematic errors. We perform a standard "Short Open Load Thru" calibration leading to reference planes located at the end of cables in the SMA connector's planes $\mathrm{R}_{1}$ and $\mathrm{R}_{2}$ (see Figure 4).

The second step consists in calibrating the measurement cell itself in order to correct imperfections due to finite conductivity of the strip and impedance mismatching. This leads to a displacement of the reference planes from $R_{1}$ and $R_{2}$ to $\mathrm{R}_{1} *$ and $\mathrm{R}_{2} *$. One can note that the change of reference is required to compare the theoretical parameters to the measured ones. This step of de-embedding is performed using conventional cascade-based method in which each section of the cell is represented by a T matrix. ${ }^{7,8}$ Figure 5 shows as a result of the de-embedding process that the theoretical response fits perfectly with the measured one in the case of the empty cell (without sample to characterize). Then the measured $S_{11}, S_{12}, S_{21}$, and $S_{22}$ parameters of the cell loaded with the material under test are de-embedded using the same
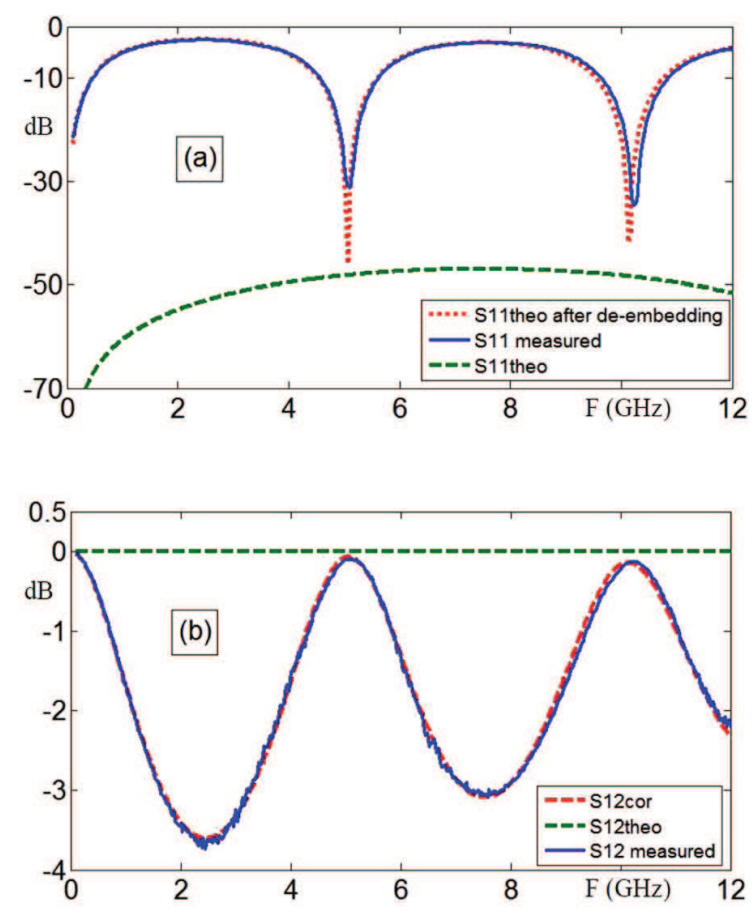

FIG. 5. Reflection (a) and transmission (b) coefficients magnitudes of the empty cell: measured (blue), theoretical before (green), and after deembedding (red). procedure in order to compare the measured and theoretical $\mathrm{S}$-parameters in the same reference plane.

The second step of experimental procedure aims to simplify the optimization procedure. The optimization consists in minimizing the error function $\mathrm{F}$ which depends on eight parameters, three for the scalar permittivity and five for the permeability tensor. Because of the large number of variables, the inverse problem can fail or give wrong results due to local minima. In order to make the optimization process more reliable, we perform the characterization of magnetic material in two steps. The first step consists in determining the permittivity only. For this, we consider that the magnetic response of ferrites in demagnetized state is mainly present in the low frequency range (less than a few gigahertz). This assumption is well suited for the compositions that we want to measure; it would be less suitable for materials exhibiting a natural gyromagnetic resonance at higher frequencies such as hexaferrites. Far enough above the gyromagnetic resonance, the material shows a purely dielectric behavior. For the permittivity determination, an asymmetric structure is not required so the measurement is done with the cell loaded only with the demagnetized ferrite $\left(\varepsilon_{2}=\varepsilon_{1}=1\right)$ which exhibits isotropic magnetic properties.

Figure 6 presents permittivity measurement results for a demagnetized commercial spinel ferrite Temex Ceramics ${ }^{\circledR}$ $\mathrm{U} 21(4 \pi \mathrm{Ms}=2400 \mathrm{G})$ in a frequency range in which the material exhibits only a dielectric behavior. Thus, the optimization
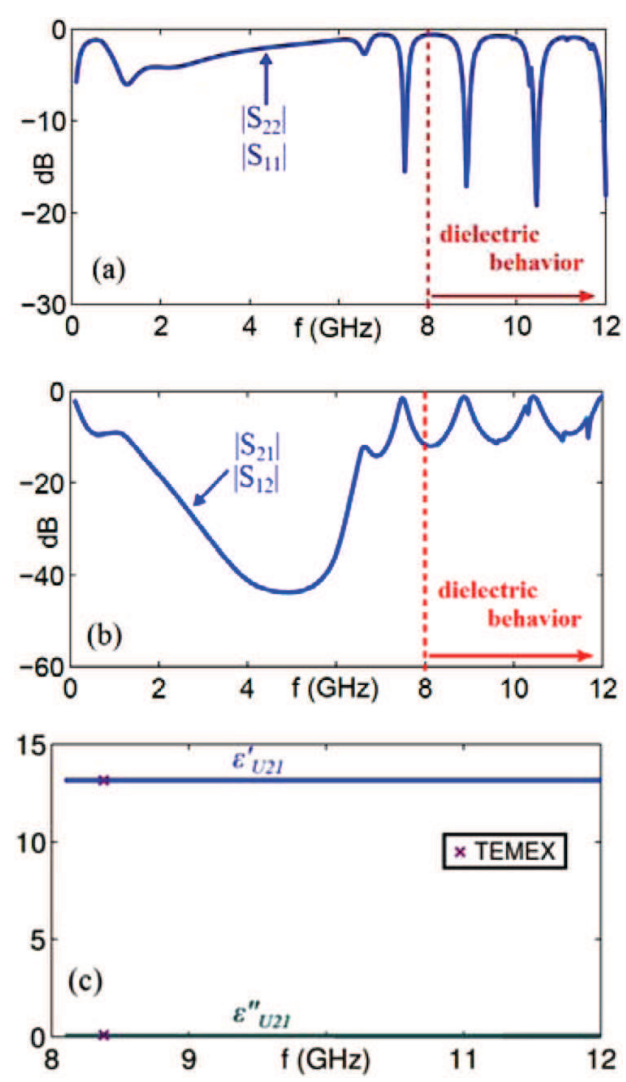

FIG. 6. Permittivity measurement of Spinel ferrite U21 in the demagnetized state (a) reflection (b) transmission coefficients magnitudes, (c) measured permittivity and provider's value. 
process is performed only in the $[8 \mathrm{GHz}-12 \mathrm{GHz}]$ range and leads to a permittivity very close to the provider's value.

One can note the presence of several dimensional resonances in the measured S-parameters due to the finite length of the ferrite sample. These resonances are error sources for a frequency by frequency optimization procedure because of uncertainties in phase measurements in the vicinity of the dimensional resonances. But they are very helpful for our wideband optimization method exploiting only the $S$-parameter magnitudes. Once the permittivity of the ferrite is determined with the reciprocal cell $\left(\varepsilon_{2}=\varepsilon_{1}=1\right)$, we assumed that the permittivity will not depend on the applied static magnetic field and will be equal to the one obtained at zero field.

Then we perform the second step of characterization in which we obtain the permeability tensor. The same ferrite is now inserted in the non-reciprocal cell $\left(\varepsilon_{2} \neq \varepsilon_{1}\right)$ and a new set of non-reciprocal S-parameters are measured. For the determination of the components $\mu$ and $\kappa$ of the permeability tensor of the magnetized ferrite, we use an optimization procedure in which the wideband permittivity spectrum is equal to the one already obtained in the first step.

\section{EXPERIMENTAL RESULTS}

For the permeability tensor measurement, the dielectric 2 was chosen in order to maximize the non-reciprocal behavior of the cell: we selected the Temex Ceramic $($ E20 $\left(\varepsilon_{2}=20.9-\mathrm{j} 0.001\right)$. Figure 7 presents the measured S-parameters of the non-reciprocal cell loaded by the commercial ferrite Temex Ceramics ${ }^{\circledR}$ Y101 $(4 \pi M s=1820 \mathrm{G})$ under a static magnetizing field of $700 \mathrm{Oe}$. The non-reciprocity of the cell is evidenced by the difference in magnitude of the transmission coefficients $\mathrm{S}_{21} \neq \mathrm{S}_{12}$.

From this measurement, the optimization procedure is performed to obtain the permeability tensor of the ferrite under test. As optimization procedures can easily lead to numerical wrong results due to local minima, we have to be very cautious on this step. We have tested several methods in our optimization code: the Matlab sub-routine lsqnonlin based on the interior-reflective Newton was compared to Matlab generic algorithm. Both lead to same results. In addition, using representative physical models for the solution of $\varepsilon, \mu$, and $\kappa$, we avoid solutions that are mathematically possible but are not physically correct. Figure 7 presents the comparison between measured and optimized S-parameters and Figure 8 presents the corresponding tensor components $\mu$

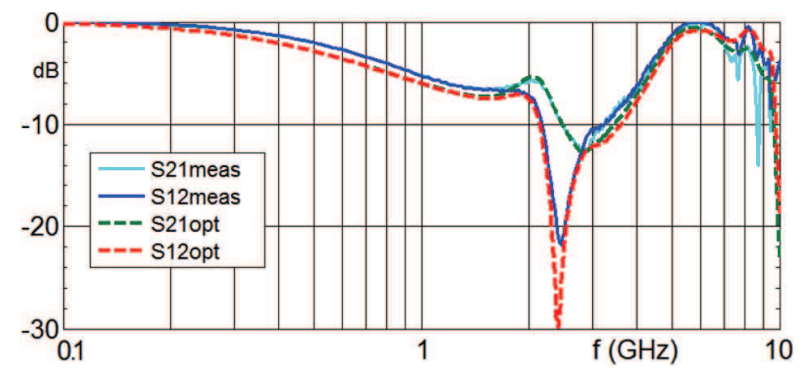

FIG. 7. Non-reciprocity of the Transmission parameter for a Y101 ferrite under 800 Oe applied magnetic field (solid line: measured parameters, dashed line: optimized parameter).

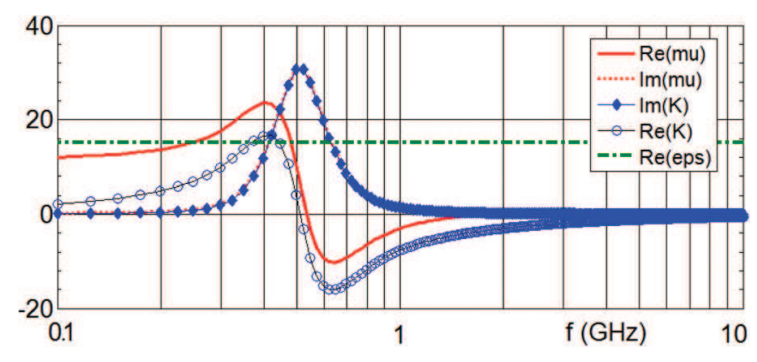

FIG. 8. Measured permeability tensor components ( $\mu$ and $\kappa$ ) spectra for the Y101 ferrite under a static applied magnetic field of 700 Oe.

and $\kappa$. These results show a good agreement over the entire frequency band, giving an error less than $7 \%$.

\section{A. Analysis}

Figure 9 shows the permeability tensor components $\mu$ and $\kappa$ extracted from the measurements of another commercial ferrite Temex Ceramics ${ }^{\circledR}$ U21 $(4 \pi M s=2400 G)$ for various applied static magnetic fields, from 0 up to 1000 Oe.

We observe a variation of the permeability as a function of the applied static field. The resonance frequency (related to the maximum of the imaginary parts of the permeability tensor components) and the static permeability (magnitude of the real parts of the permeability tensor components at low frequency) change with the applied static field. These variations are induced by the change of the internal static field which depends on the magneto-crystalline anisotropy,
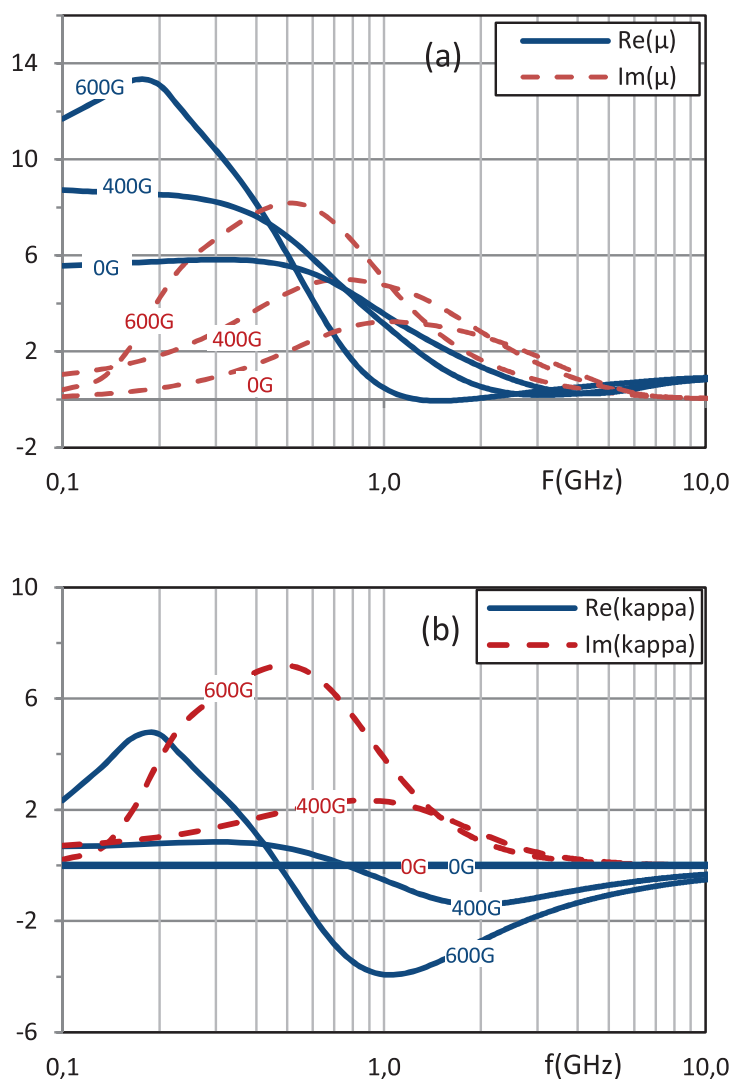

FIG. 9. Measured permeability tensor components spectra for the U21 ferrite under various applied static magnetic field up to $600 \mathrm{Oe}$. 
the applied magnetic field, and the demagnetizing fields. Initially, as the static field increases, the resonance frequency shifts toward lower frequencies and the static permeability increases [Figs. 9(a) and 9(b)]. We explain this behavior by macroscopic demagnetizing effects due to the planar shape of our sample (the dimensions of the sample are $\mathrm{x}=5 \mathrm{~mm}$, $\mathrm{z}=5 \mathrm{~mm}$, and $\mathrm{y}=1 \mathrm{~mm}$ ). Without static field applied, the magnetic moments organization preferentially lead to a distribution in the plane of the sample corresponding to a demagnetizing factor along $\mathrm{x}$ or $\mathrm{z}$ axis close to 0.15 . $^{9}$ As the magnetic field increases along y axis, moments gradually switch to a direction perpendicular to the plane of the sample corresponding to a demagnetizing factor along y axis close to 0.7 . In accordance with the Kittel law, ${ }^{10}$ the resonance frequency of the switched moments shifts to lower frequencies.
Finally, for magnetic field greater than 800 Oe, all magnetic moments have switched and see the same demagnetizing factor. Then the resonant frequency increases proportionally when the applied magnetic field increases [Figs. 10(a) and 10(b)]. We also note that the width of the absorption peak decreases as the magnetization increases due to the gradual alignment of the magnetic moments in the applied dc field direction.

As there is no magnetic standard material to validate our approach, we have compared our experimental results with those given by a well-known theoretical model: the Polder's model ${ }^{11}$ that gives accurate results in the limit case of a saturated sample. In order to account demagnetizing effects, the Polder's formulation becomes for an applied static field in the $y$ direction

$$
\begin{aligned}
& \chi=\frac{\omega_{m} \cdot\left(\omega_{o}+\omega_{m}\left(N_{z}-N_{y}\right)\right)+j \omega \alpha \omega_{m}}{\left(\omega_{o}+\omega_{m}\left(N_{z}-N_{y}\right)\right) \cdot\left(\omega_{o}+\omega_{m}\left(N_{x}-N_{y}\right)\right)-\omega^{2}\left(1+\alpha^{2}\right)+2 j \omega \alpha \omega_{o}} . \\
& \kappa=\frac{\omega \cdot \omega_{m}}{\left(\omega_{o}+\omega_{m}\left(N_{z}-N_{y}\right)\right) \cdot\left(\omega_{o}+\omega_{m}\left(N_{x}-N_{y}\right)\right)-\omega^{2}\left(1+\alpha^{2}\right)+2 j \omega \alpha \omega_{o}}
\end{aligned}
$$

where $\omega_{m}=2 \pi \gamma M_{S}, \omega_{o}=2 \pi \gamma H_{\text {app }}, \quad \gamma=2.8 \mathrm{MHz} / \mathrm{Oe}, \quad N i$ are the demagnetizing factors, $\alpha$ the damping factor and $\omega=2 \pi \mathrm{f}$.

We consider the comparison only for the limit case $\mathrm{H}_{\mathrm{app}}=1000 \mathrm{G}$ close to the saturation state. Figure $11 \mathrm{com}-$ pares the measured permeability tensor components of the U21 sample with theoretical tensor components of Polder's model filled with the provider's specifications $(4 \pi \mathrm{Ms}=2400 \mathrm{G}$ and $\Delta \mathrm{H}=290$ Oe leading to $\alpha=0.04)$ and demagnetizing factor for our samples $\mathrm{N}_{\mathrm{x}}=\mathrm{N}_{\mathrm{z}}=0.15$ and $\mathrm{N}_{\mathrm{y}}=0.7$. As one can see the measured spectra are in good agreement with the theoretical ones. We still observe some discrepancy: first a small shift in the resonance frequency which may be due to the indetermination of the true internal field in the sample and then an enlargement of measured losses which probably reflects the heterogeneity of the magnetization in our polycrystalline samples.

\section{B. Application of the method}

Beyond the dynamic permeability tensor measurement, the presented method can be a complementary alternative to conventional methods for the ferromagnetic resonance (FMR) linewidth $(\Delta \mathrm{H})$ measurements. Thus once the saturated state is reached, from the frequency spectra of imaginary permeability $\chi^{\prime \prime}(\mathrm{f})$ obtained for each value of the applied field we can extract for a given frequency the variation $\chi^{\prime \prime}(\mathrm{H})$ according to the applied static field. Compared to conventional mono frequency methods such as resonant cavities or shorted waveguide ${ }^{12}$ in which samples should be small so as not to disturb too much EM field pattern, the proposed method presents the advantage of being broadband
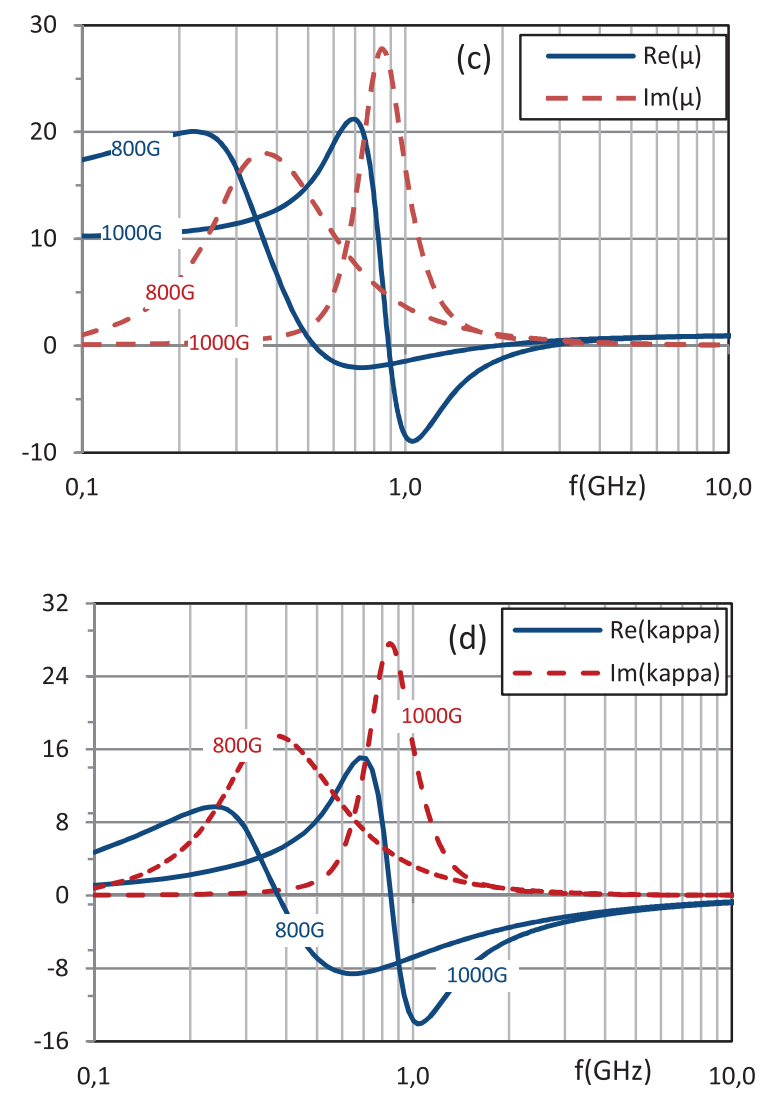

FIG. 10. Measured permeability tensor components spectra for the U21 ferrite under various applied static magnetic field up to $1000 \mathrm{Oe.}$ 

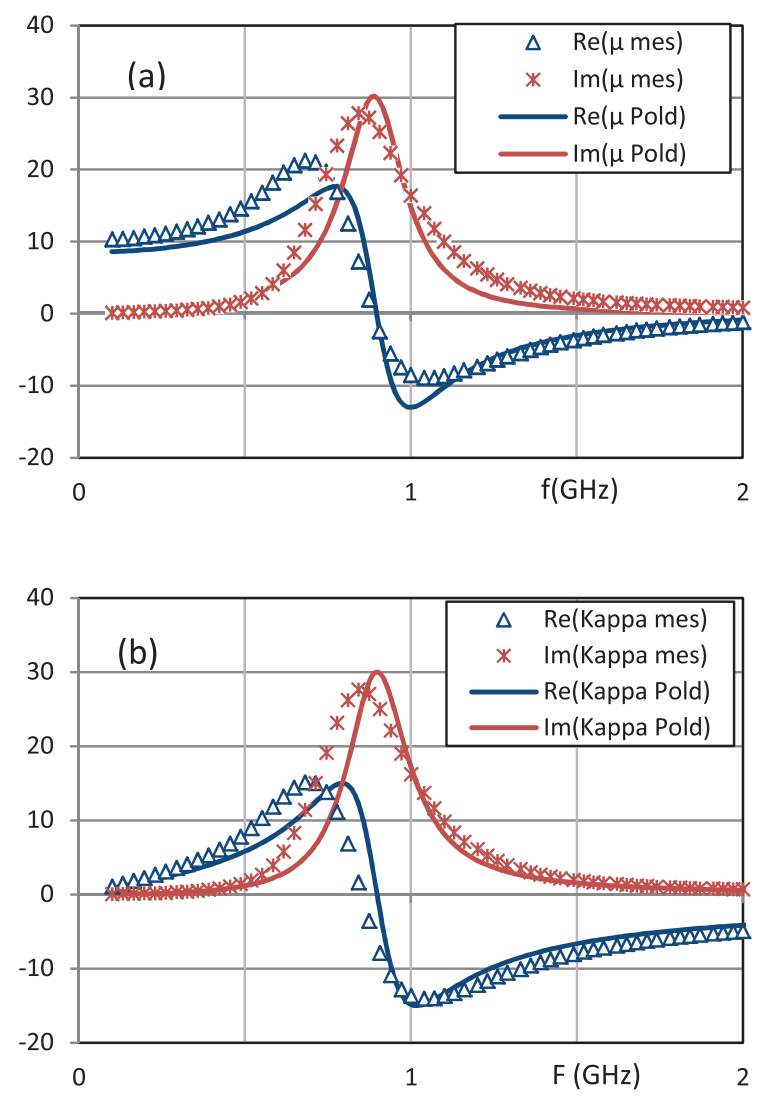

FIG. 11. Comparison between measured permeability tensor components spectra and those calculated from the Polder's formulations for the U21 ferrite under applied static magnetic field $\mathrm{H}_{\text {app }}=1000 \mathrm{Oe}$.

and using more practicable samples. In addition, compared to broadband methods such as VNA-FMR ${ }^{13}$ which rely on the quasi-static assumption and consider in the experimental setup only the transmission parameter $S_{21}$, our method is more rigorous thanks to a dynamic electromagnetic analysis taking into account higher order modes that necessary occur within the magnetized sample. Moreover we use not only the transmission coefficient $S_{21}$, but the four S-parameters to extract the permeability tensor. However, we should take care to correct the value of the internal magnetic field within the sample considering the macroscopic demagnetizing effects due to their rectangular shape. In addition, the non-ellipsoidal form may give rise to some dispersion due to the inhomogeneity of the magnetization in the sample.

\section{CONCLUSION}

The proposed method offers a new experimental tool for measuring the broadband dynamic behavior of ferrites whatever their magnetization state. The measured parameters are the permittivity $\varepsilon(\mathrm{f})$ and the permeability tensor components $\mu(\mathrm{f})$ and $\kappa(\mathrm{f})$ spectra in the $[100 \mathrm{MHz}-12 \mathrm{GHz}]$ frequency band. The data processing program relies on a full-wave analysis to take into account higher order modes (propagated in the loaded section and evanescent in the air sections of the measurement cell) and a specific broadband optimization procedure in order to avoid the uncertainties increasing of the measured phases of the S-parameters due to the dimensional resonances. In addition, the experimental setup is performed in two steps (first determining the permittivity then the permeability) in order to guarantee accurate results. Thanks to this broad-band EM characterization method which gives access directly to the constitutive parameters of magnetized ferrites appearing in the Maxwell equations, new additional procedures could be developed to determine magnetic parameters of ferrites materials such as the resonance linewidth $\left(\Delta \mathrm{H}, \Delta \mathrm{H}_{\mathrm{eff}}\right)$ not only at one frequency as it is usually done with the resonant standard method but also in a wide frequency band.

${ }^{1}$ L. F. Chen, C. Ong, C. P. Neo, V. Varadan, and V. K. Varadan, Microwave Electronics: Measurement and Materials Characterization (Wiley \& Sons, 2004).

${ }^{2}$ J. Lezaca, P. Quéffélec, and A. Chevalier, IEEE Trans. Magn. 46, 1687 (2010).

${ }^{3}$ M. Hines, IEEE Trans. Microwave Theory Tech. 19, 442 (1971).

${ }^{4}$ J. E. Lezaca, P. Quéffélec, and A. Chevalier, Int. J. Microw. Wirel. Technol. 3, 289 (2011).

${ }^{5}$ P. Gelin, P. Quéffélec, and F. Le Pennec, J. Appl. Phys. 98, 053906 (2005).

${ }^{6}$ P. Gelin and P. Quéffélec, IEEE Trans. Magn. 44, 24 (2008).

${ }^{7}$ D. Pozar, Microwave Engineering (John Wiley \& Sons, Inc., 2009).

${ }^{8}$ Agilent Technologies in Application Note 1364-1, 2004, pp. 1-24.

${ }^{9}$ A. Aharoni, J. Appl. Phys. 83, 3432 (1998).

${ }^{10}$ C. Kittel, Phys. Rev. 73, 155 (1948).

${ }^{11}$ D. Polder, Philos. Magn. 40, 99 (1949).

${ }^{12}$ I.E.T. Commission, "Measuring methods for properties of gyromagnetic materials intended for application at microwave frequencies," International Standard, IEC 60556, Section 2, 1982, p. 43.

${ }^{13}$ S. S. Kalarickal, P. Krivosik, M. Wu, C. E. Patton, M. L. Schneider, P. Kabos, T. J. Silva, and J. P. Nibarger, J. Appl. Phys. 99, 093909 (2006). 